\title{
THE DIAGNOSIS AND INVESTIGATION OF PERIPHERAL NERVE INJURIES
}

\author{
J. H. MARKS, M.B., Ch.B., Dip. Surg., Senior Medical Officer, Harmony, Rand Mines, Limited. \\ Lecture delivered to the S.A.S.P. Post Registration Course, May, 1965, \\ at the \\ Ernest Oppenheimer Hospital, Welkom.
}

\section{The Causation of Nerve Injuries}

There are many different ways in which a nerve may be traumatised-by open wounds-sustained blunt pressuretraction-chemical irritation, by misplaced injections and traction- and, moreover, a nerve may become paralysed in the various forms of peripheral neuritis.

It is rare to find a complete lesion in a closed fracture, but in compound fractures nerve injuries are common. One may see a nerve crossing a wound apparently intact, yet divided lower down.

Glass is a common cause of nerve damage and one of the reasons the Americans are said to have gone in for cardboard milk bottles is the number of cases where dairy firms have been sued as a result of children falling and cutting their hands on bottles.

According to John Chalmers, in the presence of paralysis with an open wound the chances of a nerve being severed are 90 per cent; in a closed injury the chance is 90 per cent of its not being severed.

With a severe varus strain of the knee and dislocation, one can get the popliteal nerve stretched out of existence and I have seen the ulna nerve torn in a simple fracture of the distal radius.

Peroneal palsies are common due to the stretching of the nerve across the acetabulum in fracture displacements of the hip joint and where there is a fracture in the posterior part of the acetabulum. Usually only the lateral popliteal nerve is damaged because this part is closest to the bone. Prolonged non-violent stretching of a nerve can cause paralysis. In some cases patients with fractured femora on traction show a delayed onset of foot drop. This is a true paralytic phenomenon with sensory loss and the biceps femoris is often paralysed as well.

Straight leg raising is known to throw tension on the peroneal nerve and its diagnostic value in cases of prolapsed intervertebral disc stems from this.

The cause of the paralysis is sitting up in bed with the knee straight or by excessive elevation of the straight leg. By avoiding suspending the Thomas splint above an angle of $20^{\circ}$ with the bed no further cases of foot drop have occurred.

Cast palsy is common, affecting the radial and popliteal nerves. This betokens technical errors-neglect of padding, etc.

A badly placed injection can paralyse a nerve, rarely in the axillary but commonly in the sciatic. This is surprising when one considers the number of people, semi-trained, injecting the muscle without even knowing where the axillary nerve lies.

The drunk's paralysis, due to sustained pressure whilst comatosed, affects the radial nerve or the entire plexus according to the site of pressure-over the radial nerve across a bed, or in the axilla over the back of a chair. Similar negligent paralysing effects are reported following the administration of anaesthetics, with the arm allowed to overhang the table.

\section{The Varieties of Nerve Injuries}

The generally accepted classification of nerve injuries is as follows:
Neurotmesis - a cutting - a separation of parts which may be united by fibrous tissue;

Axonotmesis - a complete division within the intact neural sheath;

Neuropraxia-or non-action of a nerve-following commotion of the fibres of the axon.

The Reaction of Nerves to Injury

Within a few minutes of interference with the conductivity of the nerve the denervated skin feels hot and dry. All reflexes are inhibited and all innervated muscles are paralysed. Atrophy is very rapid in the smaller intrinsic muscles but less so in the larger. Anomalous innervation may confuse; sensory loss follows a fairly definite anatomic pattern but overlap may occur.

There are certain autonomous zones for each nerve where overlap is not seen-these are as follows:

The Radial Nerve-a small area on the dorsum of the first interosseous space:

The Median Nerve-the volar aspect of the terminal phalanx of the index;

The Ulna Nerve - the lateral border of the 5th finger on the anterior surface;

The Popliteal Nerve-a small area on the outer aspect of the foot;

The Posterior Tibial Nerve-the sole of the foot;

The Sciatic Nerve-a combination of the popliteal and tibial areas. Other nerves will be discussed later on in this lecture.

The autonomous zone is anaesthetic; around this is an intermediate zone where sensation is reduced. This is the gross anatomic distribution of the nerve. Around this is a slightly larger zone-the maximal zone which is found when the adjacent nerves are blocked out and the nerve under examination remains intact. This is the area of overlap.

Sympathetic fibres enter the brachial plexus far proximally as post ganglionic fibres and follow sensory paths to the periphery. If a peripheral nerve is divided the sympathetic fibres are likewise divided. With paralysis due to lesions proximal to the entrance of the sympathetic fibres, the sudomotor function is intact and hence the bad prognosis is easily diagnosed.

Diminution of the blood supply of periphera. nerves causes a decrease in the rate of passage of the nerve impulse and finally to complete nerve block.

The examination of the peripheral nerve involves apart from a careful history, the general inspection of the affected member and a comparison with the normal side if possible. The examination of muscles innervated and a check of sensory defects.

\section{The Investigation of Peripheral Nerve Injury}

First one must take a reasonable history-cause of the injury-whether paralysis was immediate at the onset or delayed. One must also investigate the progress of the wound-whether sepsis occurred and also what treatment was given-physiotherapy, etc.

Pain is an important thing to discuss-the situation of the pain-radiation and time relationship to injury. 
One notes the line of entrance and exit wounds and thereby gains an idea of the nearness or risk to adjacent nerves. One should feel along the line of a nerve for-query neuroma formation. A wound in the area of a nerve often warrants exploration of that nerve.

What can the patient do? Are there any changes in the function of the part being examined?

Within a few minutes of interruption of nerve conductivity, unless above the entrance of the sympathetic fibres, whatever the cause, the denervated skin becomes flushed and dry. This is the most reliable and easily observed sign of nerve injury.

One can diagnose anaesthesia by feeling the dry skin within moments of the lesion being produced if one dries the fingers first, and also one can confidently prognosticate success in a recovering ulna lesion by feeling the moisture on the pulp of the little finger.

Many tests are based upon this sudomotor activity.

\section{The Histamine Diphosphate Test}

Histamine Diphosphate $1 / 100$ is used to test for evidence of intact post ganglionic fibres. Drop a few drops on the area to be tested and make multiple small superficial scarifications through the drops. A wheal appears if the nerve is intact or if it is severed above the ganglion station. If you can get a wheal with paralysis, the prognosis is poor because the roots are damaged.

Test all muscles innervated by each nerve in nerve lesions. Diagnose the level and also the possibility of escape of certain branches of the injured nerve.

It is important to know the anatomy and surface markings of nerves as well as their autonymous zones. An anaesthetic area is satiny smooth and the finger glides over it. The pulp atrophies and the skin lines disappear.

Guttman's Quinazoral Test has been used to demonstrate the persistence or absence of the sudomotor effects of the nerves but is messy and is not often used these days.

The test which has replaced it is called the Ninhydrin Test. There are ten different amino acids in the sweat and these stain with Ninhydrin. A moist finger passed over paper leaves a print that may be brought up by Ninhydrin many years later. It consists of punctiform representation of the glandular orifices of the sweat glands. If the fingers are wet the amino acids are high and they tend to spread over the skin and spoil the test.

\section{Details of the Test}

Roll the finger tips, which have been dried, across the paper and outline with a pencil. One should not handle the paper except at the perforated end. If the hands are moist, wash with soap and water and clean with Ether-Alcohol mixture before testing.

To encourage perspiration the patient may be given hot tea or salicylates but this is usually unnecessary.

Dip the papers in 1 per cent Ninhydrin solution (acidified with a few drops of glacial acetic acid which keeps for several months); dry paper strips-they dry quickly-and then warm them in air between 100 to $120^{\circ} \mathrm{C}$. The dots become visible. Wait a few days before fixing to get a more distinct print. Fix by dipping in 1 per cent solution of Copper Nitrate in 5/95 mixture of water and Methyl Alcohol or Acetone, acidfied with a few drops of concentrated Nitric Acid per 100 c.c.'s. The better the tactile gnosis the closer the dots.

Two point discrimination is $12-12 \mathrm{~m} . \mathrm{m}$. apart in the fingers. Tactile Gnosis does not, as a rule, return to skin grafts, but sudomotor function does return within about one or two years, even if sensation does not.

One can use this Ninhydrin Test to determine the efficiency of one's local anaesthetic and.also to get an idea of the possibility of recovering nerve function.

Where the lesion is incomplete and associated with a causalgia, sweating is excessive. It may be difficult for one to diagnose the level of the lesion where the innervation has overlapped and consequently one has to resort to the blocking of the overlapping nerve. This method of examination is known as Highets Procane Block. 5-10 c.c.'s of 5 per cent procane with Adrenalin must be injected close to the nerve.

It is also important to note the presence of associated skin changes such as oedema and cyanosis.

When you stick a pin into the finger it moves the finger and causes pain. You should use a hair-never a pin. One can often feel pin pricks with a severed median nerve because the radial and ulna nerves overlap to a certain extent.

I recommend that anyone testing peripheral nerves should use an aesthesiometer. This is a very expensive instrumentI have one here. It consists of a bicycle spoke and a piece of nylon bristle. (It is a sort of Von Frey's hair.) One should test with the side of the bristle-not with the point because then it ends to mimic the effects of the pin. One should always test for branches of the nerve that might have been intact after severance of the main trunk. If one uses cotton wool to try and test for sensation one might not even know if one is touching the skin oneself.

Tinnell's sign is the elicitation of tingling by the tapping on a nerve with open ends, but it is useless at the level of injury; if below, it shows that the nerve has crossed the level and is a good guide to the progress of recovery. If Tinnell's sign cannot be elicitated within two months of injury, below the site of the lesion, one must consider the lesion to be complete.

By testing the pulp for pin sensation with fingers flat on the table, the pin pressure is transmitted to the normally innervated skin on the dorsum of the hand, and the patient truly feels the prick. Also, if pressure moves the I.P. joints, he will be aware of deep sensation. Hold the part circumferentially in a firm grip and avoid all movement.

In muscle tests it is important to see or feel the contraction of the muscle (if one can) by testing against resisted movement or getting the patient to do some allied test.

Muscle biopsy, by showing the empty axon sheaths, allows one to differentially diagnose from the Myopathies; also the presence of the intact end organs gives one a guide to prognosis.

One can also test sensory deficiency by means of two point discrimination. Examine joints-note the passive range, attitude and gait. One should grade motor functions according to the $0-5$ system. Each muscle, as I said before, should be seen and felt to contract, if possible.

One should measure the limbs for wasting. The arm is usually measured $10 \mathrm{~cm}$. above and below the medial epicondyle, the calf at its maximum diameter and the thigh $20 \mathrm{~cm}$. below the anterior superior spine. All reflexes should be tested.

Direct electrical stimulation above the lesion may show a flicker of contraction in distally innervated muscles, in which case spontaneous recovery is likely. A hard lesion implies intraneural scar tissue and one must resect. Two forms of electricity are often used in testing. The Faradic (interrupted) and the Galvanic (constant) currents. If healthy Faradism causes a contraction as long as the current passes. The Galvanic causes contraction only when the current is made or broken. The cathode applied to the muscle gives a better response than the anode and the induction current is more effective than the break. In degeneration of the nerves the anode may give a brisker contraction than the cathode and the reaction may be sluggish. The Faradic current acts on the Nerve. The Galvanic on the Muscle.

Reaction of Degeneration-No response to Faradism.

Galvanic Reaction is to a lesser current but the muscle contracts sluggishly and the anode contraction is greater than the cathode. Reaction of degeneration takes over a week to develop. Usually the muscles return to power before the nerves respond to electrical stimulation. These test are not of much use.

Two ordinary hypodermic needles attached to two $1 \frac{1}{2}$ volt dry cell batteries with a polarity reversal switch are all one really needs to test nerve conductivity. I show here the apparatus we use at Harmony kindly devised by Mr. V. S. Gore of this mine.

In a recent complete division of a sensory nerve the skin 
is pinkish because of vascular dilation (depending on the is pision of sympathetic fibres). A few days later the skin divis may show fine scaling. Where the lesion is older the skin is bluish mottled and dry. Where the lesion is irritative the skin may be red and perspiring.

Muscle power is graded on the 0-5 system.

0 is no function;

1 is a flicker only;

2 is movement with gravity eliminated;

3 is contraction against gravity only;

4 is contraction against moderate resistance;

5 is normal power.

Pain sensation is often readily tested with a pin. Ask if he feels sharp or blunt using opposite ends.

Hot or cold sensation can be tested using test tubes, with hot water and ice but is usually not needed.

\section{The Limbs as a Whole}

Muscles that are innervated by the nerve under examination must all be checked-seen or felt to contract-and their strength investigated according to the 5-0 method. When it can be shown that a paralysed or weakened muscle has regained function or strength it is a definite sign that there has been nerve recovery. Where one of the muscles that are innervated below the site of a lesion is functioning it proves the incompleteness of the lesion. Also where the level of a lesion is in doubt the decision as to which of its muscles are and which of its muscles are not functioning will aid in locating the level of section. In the time allotted to me I now briefly wish to mention the methods of checking up on special nerves. An easy way of ruling out an injury to a nerve in the upper limb is to test the sensation of the automymous zones of the median and ulna nerves by means of a Von Frey's hair and compare with the opposite side. The anterior aspect of the index and little fingers - the radial nerve is tested by getting the patient to open the hand widely and extend the digits.

\section{For the Lower Limb}

Test the sensation between the Hallux and the second toes and compare. This tests the tibial nerve. If he can extend the great toe strongly, the perioneal nerve is not affected.

A limb may take up a characteristic position after injury. The Policeman's tip of the Erb Duchenne paralysis and the radial nerve wrist drop or the Benediction attitude of the median nerve when the hand is outstretched due to the lack of flexion of the index.

The skin is important-in a recent injury with complete severance of sympathetic fibres the skin is flushed due to dilation and often a fine desquamation occurs after a few days. After several days the skin is dry and mottled. Trophic ulceration may occur. With an irritative lesion the skin is moist and glossy.

\section{Muscle Examination}

It is most important to know what muscles are involved in any lesion-the presence of function in the territory of an involved nerve is of note.

After about 4-6 weeks the characteristic deformities of the various lesions are apparent.

It is wise to look for tendon damage as a possible cause of muscle failure that might mimic paralysis.

Assess the range of joint movements. It is of no avail to repair nerves supplying muscles that work on "frozen" joints.

Skin temperature is controlled by the rate of blood flow in the skin vessels. The patient's disabilities are more important than the objective findings. A paralysed limb becomes warmer in hot and colder in cold surroundings than the normal limb. After successful suture there is often a sensation of increased warmth in the part. The return of sweating is a late feature and indicates extensive regeneration. With paralysis the nails are brittle and tend to incurving.

Trophic changes are particularly common in the skin of the median and posterior tibial distribution.

\section{More Detailed Description of the Various Nerves, etc.}

The innervation of movement is segmental in development. Any movement is innervated by two adjacent segments and the antagonistic movements by two adjoining segments. The entire group of movements of a joint is controlled by four segments in series and, passing distally, the four seried segments start one segment lower.

The spinal centre for the hip is thus Lumbar 2, 3,4 and 5; for the knee Lumbar $3,4,5$ and $S .1$; and for the ankle Lumbar 4, 5, S.1 and 2. The anterior muscles are controlled by the upper 2 segments, thus in the hip-Lumbar 2 and $3-$ control flexion, adduction and medial rotation; Lumbar 4 and 5-the opposite; extension, abduction and external rotation. In the knee, the extension is Lumbar 3 and 4; flexion is Lumbar 5 and S.1. In the ankle, dorsi flexion is Lumbar 4 and 5, and planti flexion is S.1 and 2 .

To remember the innervation of a muscle, remember its prime function. In the upper limb-alas for memory-the rule is not 100 per cent reliable. Shoulder-abduction and external rotation is C.5; adduction and internal rotation is C.6, 7 and 8. The elbow has the same 4 segments. Flexion is C.5 and 6 and extension is C.7 and 8 .

Pronation and supination is C.6; wrist movement is C.6 and 7; Thumb and finger movement is C.7 and 8 ; intrinsic mechanism of the hand is T.1.

One remembers the innervation of Reflexes as above. The reflex that extends the knee-the knee jerk is thus L. 3 and 4. The reflex that plantiflexes the foot is thus S.1 and S.2.

Either get the patient to perform actions against resistance or to resist movement in the opposite direction. See and feel the muscle bellies.

The Brachial Plexus: often injured in birth accidents.

Two main types apart from a total paralysis. The Erb Duchenne-upper roots C.5 and 6 affecting mainly the upper arm. The arm hangs limply by the side and the wrist is flexed and the forearm pronated. "The Policeman's Tip" position.

The Klumpke Paralysis: C.8 and T.1.

A paralysis of the muscles of the hand and true claw. Loss of sensation along the ulnar side of arm, forearm and hand and a Horner's syndrome is seen. Claw hand is seen in lesions of the inner cord of the Brachial Plexus-Syringomyelia-in combined median and ulna nerve lesions at the wrist and also after Volkman's ischaemic contracture.

The Accessory Nerve: C. 1, 2, 3 and 4-the supply of the sterno-mastoid (1) and the trapezius muscles.

(1) Turn the head to the side of the lesion and get him to resist the movement of the chin. See or feel the muscle contract.

(2) Get him to shrug his shoulders and brace them back, against resistance. The paralysed side does not shrug and if of longish duration the shoulder is flattened. Can feel as well as see the contraction.

We mention here Horner's syndrome which is caused by interference with the cervical sympathetic arising per T.1 and T.2. It consists of a small pupil (myasis); a dry skin (anhidrosis); a partial ptosis of the upper eye lid and a slight sinking in of the eyeball-enophalmos. It also carries a serious prognosis in upper cord lesions.

\section{The Cervical Rib Syndrome}

Mostly a cervical rib gives rise to no symptoms at all. Where it does have effects these are vascular or neurogenic. The vascular are those of defective circulation extending to gangrene. The nerve symptoms are chiefly originated in the T.1 segments via the ulna nerve with wasting of the small muscles of the hand and the Thenar Eminence. Sensory changes are not common.

The Axillary Nerve (Circumflex) C.5 and 6 supplies the deltoid and may be involved in injuries of the shoulder. There is a small area of anaesthesia over the deltoid and abduction of the arm is impossible. Test by abducting the the arm between $15^{\circ}$ and $85^{\circ}$ and get him to try and maintain the position against resistance. Can see and feel the muscles contract.

The Dorsal Scapular Nerve (C.5) is the nerve to the 
rhomboids. Put the hands on the hip and brace the elbow back against resistance. Can see and feel the muscles contract.

The Supra Scapular Nerve: C.5 and 6 supplied the superior and inferior scapular muscles. There is wasting above and below the spine of the scapula. The muscles are tested separately. The arm hangs by the side and when he tries to abduct it can feel the supraspinatus contract.

To test the infraspinatus bend the elbow to a right angle and externally rotate the shoulder against resistance.

The Long Thoracic Nerve: C.5, 6 and 7, supplies the subscapularis. When the patient pushes against some immovable object (as a wall) the scapulae stay close to the chest wall unless the muscle is paralysed when one gets winging of the scapula.

The Long Subscapular Nerve (or Thoracodorsal nerve) derived from C.6, 7 and 8 supplies the latissimus dorsi. Grasp the muscle and let him cough. The muscle can be felt to contract under the fingers. Little disability is caused by dysfunction of this muscle.

The Ulna Nerve: C.8 and T.1-may be paralysed due to disease (Leprosy) or trauma.

The interossei waste and the metacarpals stand out. Clawing occurs if the lesion is at the wrist level. The little finger is abducted due to the unopposed avion of the extensors. In clawing the long extensors extend the M.P. joints. The interphalangeal joints are extended by interossei and the lumbricals and these are paralysed. The unopposed long flexors pull the fingers into a claw. Obviously this cannot happen if the long flexors are paralysed by an high lesion.

In ulna loss one may only get skin sensation loss on the anterior aspect in the midline of the little finger. By putting the 5 th finger apart and pushing them together, you will be able to demonstrate even the most minor degrees of ulna paralysis, as in ulna neuritis. One can do the same with the index finger for the first interrosseous. When testing the interrossei get him to move his fingers from side to side, holding the other fingers apart, but make sure that the fingers are fully extended or the long flexors will confuse the issue by mimizing the movement.

The Card Test for the Interossei

See that the fingers are kept straight and not flexed at all and get him to try and grip a card between the fingers and resist fully. The card slides out.

The Adductor Pollicis-Ulna nerve

Get him to hold a card between index and thumb of each hand with the thumb straight. Due to weakness of the adductor the flexor pollicis longus (median nerve) takes over and flexes the I.P. joint.

\section{The Radial Nerve}

This supplies the Triceps--all extensors of the forearm. and the Supinator. It is derived from C. 5, 6 and 7 and often T.1. At the elbow it divides into two branches-The Posterior Interrosseous nerve (muscular) and the Superficial Radial (sensory). If the lesion is above the upper/middle third of the arm the Triceps are spared. If above the elbow the Brachioradialis is spared. The characteristic wrist drop is often missed unless the elbow is bent and the forearm pronated.

Where the posterior interrosseous nerve is affected there is no wrist drop but an inability to extend the M.P. joints. This is a serious disability for pianists, typists, etc.

The Median Nerve (C.6, 7, 8 and T.1) may be injured at either elbow or wrist. If divided in the region of the elbow there is an inability to flex the thumb. The thenar muscles waste and the palmar surface of the thumb rotates to face forwards - the Ape-like hand.

\section{Tactile Gnosis-or Touch Sight}

With impaired sensibility of the median nerve, the patient picks up articles betwen his thumb and his ring and little fingers, instead of between index and thumb. Let him pick up a number of articles with each hand, under vision, and notice how he uses his hand. Get him to identify these articles blindfolded-screw, a cap, a paper clip and so on. One must have tactile gnosis to do fine work-to use a screwdriver, wind a watch, etc. Areas deficient in tactile gnosis are, of course, obviously deficient in the ability to perspire. The loss of tactile gnosis is the crippling effect of a median lesion.

Get him to clasp his fingers together. The index stands out due to paralysis of the flexor. Test the flexor pollicis longus - fix the M.P. joint of the thumb and get him to bend the terminal phalanx. At the wrist the abductor pollicis brevis will be affected. Get the patient to place his hands palms down with the index and thumb touching in the Oriental Prayer position and raise the hands before the face. The tip of the affected thumb touches the base of the palmar aspect of the pulp and not the tip. The abductor pollicis brevis is not working. The sensory loss may be the classical lateral $3 \frac{1}{2}$ digits but may only be the autonymous zone of the pulp of the index.

The Carpal Tunnel Syndrome-A progressive weakness of the finer movements of the hand-hyperaesthesia of the median nerve distribution and an aching pain that may extend up as far as the shoulder. The thenar eminence muscles waste and the abductor pollicis brevis and the opponens pollicis atrophy.

If one gets the patient to keep his wrists flexed for a minute the pain is exaggerated. Nearly 90 per cent of the cases get pain on percussion of the median nerve at the wrist.

The Lower Limb-Nerve damage is common in the gluteal region, the Inguinal region and the knee and ankie.

The Femoral Nerve (L.2, 3 and 4)

Any of its branches to the quadriceps may be severed See if he can extend the knee-feel the heads contract. Anaesthesia in the anterior aspect of the thigh and down the middle of the leg may be seen.

\section{The Obturator Nerve (L.2, 3 and 4)}

On his back with the knee extended feel the muscles attempting to adduct the limb against resistance.

The Sciatic Nerve is often involved in disc lesions. The history is important-injury or strain must be described fully bearing in mind that trivial injuries may cause marked disc lesions. The history of the pain starting in the buttock with spreading down to the thigh and foot is important. Note the posture-the scoliosis-the semi-flexed position when standing.

The thigh is flexed and the patient lies with the heel as high up on the opposite thigh as can be comfortably placed. Now press the knee down and out in the bed. If there is pain before the knee touches the bed this is not a nerve pain but due to arthritic changes in the hip on sacro-iliac joints. The sciatic nerve is really two nerves in a single sheaththe common peroneal and the tibial nerves. joint.

Total Sciatic Palsy: Paralysis of all muscles below the knee

\section{The Inferior Gluteal Nerve-from L.5 and S.1 and 2}

Get the patient to lie on his face and extend the thigh. Feel the gluteus maximus muscle contract.

The Superior Gluteal Nerve (L.4 and 5 and S.1) supplies the Gluteus Medius and Minimus and the Tensor Fasciae Latae.

Can be tested by getting the patient to stand and raise the leg. Where the gluteus medius is paralyzed there is a drop of the hip of the affected side. This causes a characteristic gluteal gait. The drop of the hip is the Trendallenburg Test.

If the patient lies supine with the thigh straight and tries to abduct the thigh against resistance the muscle bellies can be seen and felt. If he lies prone with the knee bent at a right angle and attempts to internally rotate the leg against resistance one can see and feel the muscles contract.

Common Peroneal Nerve: This can be rolled between the fingers where it crosses the head of the fibula. In neuritis it is very tender. Paralysis of the nerve causes foot drop. 
This small book should be of great assistance to the student in understanding the abnormalities which arise from injury to the cerebral nervous system and also to the physiotherapist in assessing and planning treatment for patients.

M. HORSLEY, M.C.S.P.

HAVING A BABY. By J. F. Robinson, M.B., Ch.B. 3rd Edition. Publishers: E. \& S. Livingstone Ltd., 15/17 Tevoit Place, Edinburgh. 8s. 6d. net, England.

This is a wonderfully written book for "young marrieds". It deals clearly and concisely with problems which many do not like to discuss. Provided that the reader has adequate ante- and post-natal physiotherapy training this book cannot fail to be of considerable help.

The ante- and post-natal exercises mentioned are well chosen, but because of the danger of persistent sacro-iliac strain occurring post-natally it is generally believed that "double leg-raising" is contra-indicated in all ante- and post-natal work. It is particularly dangerous to include post-natally "double-leg raising" as the abdominal muscles are less able to control the lumbar lordosis which occurs in most cases. Although the author does indicate this exercise to be performed later in the peuperium, he does not give any earlier progression of abdominal exercises.

BRENDA KASTELL, M.C.S.P.

REHABILITATION OF THE LOWER LIMB AMPUTEE.

By W. Humm, M.S.R.G. 84 pages, 40 illustrations, Price

18s. Publishers: Bailliere Tindall and Cassell Ltd., 7 and 8

Henrietta Street, London, W.C.2.

This comprehensive book comes to the aid of the rehabilitation therapist who is entrusted not only with the reambulation of the amputee, but also with the psychological adjustment of the patient to his artificial limbs.

The book is divided in two parts: the pre-prosthetic phase, and the prosthetic and late phase treatment, Psycholological problems, stump exercise routine, and stump bandaging are the main items dealt with in the first part. All stages of re-ambulation for single and double amputees, as well as the care of stumps and prostheses are extensively described in the second part of the book.

The accent throughout is on the practical approach. Our well-trained physiotherapy students, and those already qualified will find that the book is adding to their thorough grounding those practical hints which can only come from an expert with great experience. The author is a perfectionist who does not accept a second-class result. He knows well that the beginning of prosthesis-walking are not easy for the patient and that the amputee tries to get away with any amount of easier, but incorrect movements, which produce an unsightly gait, early fatigue and harm to the equipment for all of which in the end nobody but the instructor would be blamed. The attention of the reader is directed towards such possible mistakes by the amputee and he is shown how to avoid them. Every point is made clearly by way of succinct description, clever line drawings and carefully selected photographs. Being a guide to rehabilitation, the book does not stop at re-ambulation, but adds to this in the final stages, under separate headings for unilateral and bi-lateral amputees, such functional activities as stair-climbing, getting on and off public transport, falling and getting up from the floor and numerous other moves.

The book should be of great benefit to many who are concerned with the re-ambulation of amputees of all agegroups.

A. ROTHBERG

CLAYTON'S ELECTROTHERAPY AND ACTINOTHERAPY. A Textbook for Student Physiotherapists. 5th Edition, by Pauline M. Scott, M.C.S.P., T.E.T., T.M.M.G. School of Physiotherapy, Kings College Hospital, London. Publishers: Bailliere, Tindall and Cassell, 7 and 8 Henrietta Street, London, W.C.2. 390 pages, 205 illustrations, 35s. net. 1st September, 1965 publication date.

(To be reviewed later.)

Diagnosis and Investigation of Peripheral Nerve Injuries. - Continued from page 6 .

There is a highstepping flapping gait due to inability to dorsifiex the ankle.

The Tibial Nerve

Paralysis here leads to paralysis of all the muscles of the calf and the foot is pulled up by the Peroneal group so that he stands on his heel. The ankle Jerk is absent because the muscle concerned is paralysed.

\section{A. C. Miller \& CO. ORTHOPAEDIC MECHANICIANS}

Manufacturers and Suppliers of:

ORTHOPAEDIC APPLIANCES, ARTIFICIAL LIMBS, TRUSSES, SURGICAL CORSETS, URINALS, ARCH SUPPORTS, COLOSTOMY BELTS, ELASTIC STOCKINGS, ANKLE GUARDS, WRIST GUARDS, ELBOW GUARDS, KNEE GUARDS, LIGHT DURAL CRUTCHES FOR CHILDREN, WOODEN CRUTCHES, AND METAL ELBOW CRUTCHES.

Phone 23-2496 P.O. Box 3412 312 Bree Street, Johannesburg 